\title{
La pandemia de COVID-19 ha impulsado el manejo de la diabetes vía telemedicina
}

\section{Telemedicine diabetes management has been boosted by COVID-19 pandemia}

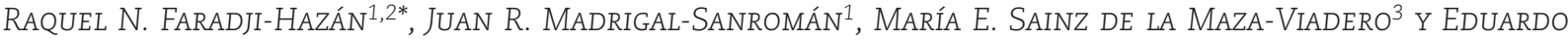 \\ MÁRQUEZ-RODRÍGUEZ ${ }^{4}$
}

'Endocrinología y Diabetes, Clínica EnDi, Ciudad de México; ${ }^{2}$ Endocrinología y Diabetes, Centro Médico ABC, Ciudad de México; ${ }^{3}$ Educación en Diabetes, Clínica EnDi, Ciudad de México; ${ }^{4}$ Dirección médica, Instituto Jalisciense de Metabolismo, Guadalajara, Jalisco, México

\section{RESUMEN}

La pandemia por enfermedad por coronavirus 2019 (COVID-19) ha venido a cambiar radicalmente la atención médica. La diabetes se ha identificado como factor de riesgo que aumenta la mortalidad, pero un buen control glucémico (glucosa entre 70 y $180 \mathrm{mg} / \mathrm{dl}$ ) se asocia con disminución significativa de la gravedad de la enfermedad y la mortalidad. Las personas que viven con diabetes deben mantener aislamiento físico y al mismo tiempo su seguimiento médico, mejorando su control glucémico para disminuir riesgos. Es por esto que la atención médica a distancia es tan importante en estos momentos. La telemedicina interactiva permite a médicos y pacientes comunicarse en tiempo real, por medio de una videoconferencia. Esto ha sido posible gracias al uso difundido de expedientes médicos electrónicos y de teléfonos inteligentes en la población. La endocrinología, y en específico la diabetología, son especialidades ampliamente compatibles para el manejo con telemedicina. En este artículo se describe la historia de la telemedicina, su actualidad, en qué consiste y qué plataformas existen para su aplicación, qué pasos se deben seguir en una teleconsulta, ventajas y desventajas, su aplicación en pacientes con posible COVID-19, la evidencia científica del uso de la telemedicina en el manejo de la diabetes y el marco legal de ésta.

Palabras clave: Diabetes. Telemedicina. COVID-19. Pandemia.

\begin{abstract}
COVID-19 pandemic has come to change medical attention radically. Diabetes has been identified as a risk factor that increases mortality. Good glycemic control (70 to $180 \mathrm{mg} /$ $\mathrm{dL}$ ) is associated with a significant reduction in the severity of the disease and its mortality. Persons living with diabetes must maintain physical distancing, and at the same time, keep their medical follow-up, ideally improving their diabetes management to reduce risks. For these reasons, remote medical attention is so important now. Interactive telemedicine allows physicians and patients to communicate in real time via videoconference. This has been possible thanks to the widespread use of electronic medical records and smartphones in the general population. Endocrinology, and specifically, Diabetology, are specialties that are compatible with telemedicine. In this article we describe the history of telemedicine, its state of the art, what it is, which platforms exist, which steps should be followed in a remote consultation, advantages and disadvantages, its application with COVID-19 patients, the scientific evidence of the use of telemedicine in diabetes management, and the legal framework.
\end{abstract}

Key words: Diabetes. Telemedicine. COVID-19. Pandemic.

\section{Correspondencia:}

*Raquel N. Faradji-Hazán

E-mail: rfaradji@gmail.com
Fecha de recepción: 11-05-2020

Fecha de aceptación: 13-05-2020

DOI: 10.24875/RME.20000038
Disponible en internet: 11-06-2020 Rev Mex Endocrinol Metab Nutr. 2020;7:87-95

2462-4144 / ( 2020 Sociedad Mexicana de Nutricion y Endocrinologia, AC. Publicado por Permanyer. Éste es un artículo open access bajo la licencia CC BY-NC-ND (http://creativecommons.org/licenses/by-nc-nd/4.0/). 


\section{INTRODUCCIÓN}

La pandemia por enfermedad por coronavirus 2019 (COVID-19), causada por el coronavirus 2 del síndrome respiratorio agudo grave (SARS-CoV-2), ha venido a cambiar radicalmente la atención médica. La diabetes se ha identificado como un factor de riesgo importante para mortalidad. La COVID-19 aumenta esta un $2.3 \%$ en personas sin diabetes frente a un $7.3 \%$ en personas con diabetes. En un artículo reciente, se ha demostrado que un buen control glucémico (medido como glucosa entre 70 y 180 $\mathrm{mg} / \mathrm{dl}$ ) se ha asociado con una disminución significativa de la gravedad de la enfermedad y de la mortalidad (hazard ratio: 0.14$)^{1}$.

Las personas que viven con diabetes, por un lado, deben mantener el aislamiento físico, quedándose en casa para evitar el contagio, pero por el otro, deben mantener su tratamiento médico $y$, de preferencia, mejorar su control glucémico para disminuir sus riesgos. Es por esto que la atención médica a distancia es tan importante en estos momentos. Desafortunadamente, la mayoría de los institutos de salud y centros médicos nacionales del país se han convertido en centros para atención de pacientes con COVID-19, lo cual ha dejado a los pacientes que viven con enfermedades crónicas sin su atención médica regular. En esta coyuntura, la atención no hospitalaria cubre dos propósitos: cuidar el control metabólico y servir de filtro, para evitar acudir a los centros hospitalarios de forma innecesaria.

Justo en el momento en que es más importante llevar un buen manejo de la diabetes y cuando los pacientes tienen más ansiedad por su condición, se podría perder el contacto con su equipo de salud. En este entorno la telemedicina se convierte en una gran herramienta para limitar este problema.

\section{RESEÑA HISTÓRICA SOBRE} TELEMEDICINA

La primera referencia de la telemedicina data del siglo xx: en 1905 Eithoven realizó la transmisión de la señal de un electrocardiograma a su laboratorio. Posteriormente, en 1951, se realizó la primera videoconferencia, en la Feria Mundial de Nueva York, la cual dio pie para el desarrollo de esta nueva tecnología. En 1985 se reportaron las primeras consultas de telemedicina a los habitantes de la tribu Pápago localizados en las afueras de Tucson, Arizona, mediante el proyecto STARPAHC (Space Technology Applied to Rural Papago Advanced Health Care), y en ese mismo año, la NASA (National Aeronautics and Space Administration) proporcionó atención por medio de telemedicina en el terremoto de la Ciudad de México².

La Organización Mundial de la Salud (OMS) definió en 1998 la telemedicina como: «el suministro de servicios de atención sanitaria en los que la distancia constituye un factor crítico, por profesionales que apelan las tecnologías de la información y comunicaciones con el objetivo de intercambiar datos para hacer diagnósticos, mejorar e indicar tratamientos y prevenir enfermedades y heridas, así como la formación permanente de los profesionales de atención de salud y en actividades de investigación y de evaluación, con el fin de mejorar la salud de las personas y de las comunidades en que viven». En mayo de 2005 la OMS emite una resolución sobre e-salud, en donde reconoce el impacto que el uso de las tecnologías de la información y las comunicaciones (TIC) representa para brindar servicios de atención médica, la salud pública, vigilancia epidemiológica, educación en salud, investigación y para las actividades y disciplinas relacionadas ${ }^{3}$.

Ante la pandemia de influenza H1N1, en el año 2009, resurgió el concepto del uso de TIC, haciendo evidente la necesidad de creación de entornos multimedia de comunicación mediante cibersalud, salud móvil y telemedicina; sin embargo, el medio más utilizado fue el teléfono y herramientas recién desarrolladas como Skype y el chat en internet. Desgraciadamente la mayoría de los avances en TIC tuvo lugar en los países desarrollados, en el resto del mundo fueron subutilizados. Posiblemente, uno de los efectos más significativos de las TIC ante la pandemia de la influenza fue el establecimiento de entornos educativos e informativos, dada la posibilidad que ofrecieron para flexibilizar el tiempo y el espacio donde se intercambia la información ${ }^{4}$. 
En México, el Plan Nacional de Desarrollo 2007-2012 fue creado con el objetivo de brindar igualdad de oportunidades con servicios de salud eficientes, tomando en cuenta las tecnologías del área de la salud, ampliando la cobertura de los servicios por medio de unidades móviles y el impulso de la telemedicina. Este plan se implementó con la idea de ampliar los servicios a la población sin posibilidades económicas por lo que, dentro del Programa Sectorial de Salud, se intenta ampliar el acceso y mejora de la calidad, la efectividad y la continuidad de la atención médica mediante el uso de telesalud. Un ejemplo de telemedicina implementada en el 2008, fue Telemedicina Sonora, programa que forma parte de la Dirección de Servicios de Salud de la Persona, en la modalidad de teleconsulta, ante la necesidad de proporcionar atención médica especializada a la población que radica en localidades alejadas del Estado, con la finalidad de mejorar sus condiciones de salud, y ante la urgencia de optimizar los recursos tanto humanos como financieros mediante el uso de nuevas tecnologías de información y telecomunicaciones. Dicho programa sigue funcionando con cuatro centros de telecomando y cinco unidades periféricas de diagnóstico y referencia, proporcionando servicios de consulta de especialidad en las áreas de: psiquiatría, oftalmología, cirugía, dermatología, medicina interna, pediatría, otorrinolaringología, y traumatología y ortopedia. Con Telemedicina Sonora se proporcionaron un total de 2,160 consultas de especialidad de abril del 2008 a mayo del 2010, además de capacitación a personal administrativo del Seguro Popular, con lo cual hubo ahorro considerable de recursos, principalmente de viáticos ${ }^{5}$.

\section{ACTUALIDAD}

Actualmente nos enfrentamos a la pandemia de COVID-19, y tanto médicos como profesionales de salud nuevamente han adoptado estrategias a distancia para evitar reuniones presenciales con los pacientes y población en general. La telemedicina ha sido posible gracias al uso difundido de expedientes médicos electrónicos y de teléfonos inteligentes en la población.
En China, después de que la pandemia emergiera en diciembre del 2019, se instó a la población a buscar atención digital mediante telemedicina en vez de exponerse de manera presencial, y gracias a las tecnologías existentes, los médicos pudieron consultar a cientos de pacientes por día. En Italia, en alrededor de 20 regiones, se han implementado sistemas de telemedicina, aunque se reportó insuficiencia en la capacidad debido al incremento en la demanda hasta en un $90 \%$. EE.UU., en particular, ha sufrido una transformación nunca antes vista en cuanto a los servicios de salud, tomando como ejemplo a China: los sistemas de salud Medicare \& Medicaid Services, los cuales forman parte del gran sistema de salud pública, autorizaron más de 80 servicios vía telemedicina, asegurando acceso a consulta médica y otros servicios de salud mientras las personas se mantenían seguras en casa. Sin embargo, la pregunta real es ¿se mantendrán estas medidas a distancia después de la pandemia? Con los teléfonos móviles usados de manera global, se adoptan tecnologías virtuales de manera más fácil, siendo una oportunidad para implementar la telemedicina por esa vía6

La pandemia por COVID-19 nos ha enseñado que no podemos esperar a tener una transición a un nuevo modelo de salud que integre por completo a la telemedicina. El cambio está ocurriendo ahora. La telemedicina nos permite abordar y dar continuidad a pacientes que sufren de enfermedades crónicas principalmente, y que no pueden esperar a ser atendidas durante emergencias nacionales.

\section{¿QUÉ ES LA TELEMEDICINA?}

La telemedicina es la prestación a distancia de servicios clínicos de salud gracias a la infraestructura de telecomunicaciones. En general, «busca mejorar la salud de un paciente, permitiendo la comunicación interactiva en tiempo real entre el paciente y el médico o profesional a distancia. Esta comunicación electrónica conlleva el uso de equipos de telecomunicaciones interactivas que incluyen, como equipamiento mínimo, audio y vídeo» ${ }^{7,8}$.

La telemedicina se puede utilizar para tres situaciones específicas: 
- La monitorización de pacientes a distancia. Ocurre cuando tenemos dispositivos que recopilan datos sobre registros de glucosa, presión arterial y otros signos vitales, y estos pueden ser enviados al equipo de salud. Idealmente deberían incorporarse directamente al expediente médico electrónico. Este tipo de registros puede ser analizado posteriormente de manera asincrónica.

- La tecnología de almacenamiento y envío. Se utiliza cuando se toman imágenes de rayos $\mathrm{X}$ o retinografías, por ejemplo, en un centro ambulatorio y son enviadas inmediatamente a un centro especializado para su interpretación. Ya interpretadas, son incorporadas al expediente electrónico del paciente.

- La telemedicina interactiva. Permite a médicos y pacientes comunicarse en tiempo real, por medio de una videoconferencia. Al ser en tiempo real, esto ocurre de manera sincrónica, y se toman acuerdos y decisiones médicas en el momento de la consulta.

Es importante mencionar que, en la medida de lo posible, se debería contar con un formulario de valoración inicial digital, que el paciente complete en línea previo a la cita, para tener su nombre completo, fecha de nacimiento, datos de contacto, y de preferencia una ficha médica similar a la que se cuenta en el expediente médico electrónico (antecedentes personales patológicos, no patológicos, estilo de vida, etc.), para así facilitar la información y hacer más eficiente y efectiva la consulta.

Idealmente, en preparación para el día de la consulta se debe contar con la lista actualizada de medicamentos que toma el paciente, los resultados de análisis de laboratorio, los registros obtenidos de diversos dispositivos, como los de glucosa (ya sea realizados manualmente o mediante una aplicación del glucómetro), del monitoreo continuo de glucosa y de las microinfusoras y los signos vitales del paciente (peso, presión arterial, frecuencia cardiaca, saturación de oxígeno y temperatura).

\section{¿QUÉ ES LA CONSULTA PSOAP?}

Debido a que la teleconsulta, en la mayoría de los casos, va a carecer de la exploración física, para
Tabla 1. Componentes del método PSOAP para la consulta telemédica

\begin{tabular}{ll}
\hline Método PSOAP para consulta a distancia \\
\hline Paciente & - Características clínicas del paciente \\
& - Lista de problemas \\
& - Lista de medicamentos \\
\hline Subjetivo & - Síntomas \\
& - Necesidades del paciente \\
\hline Objetivo & - Exploración física (limitada) \\
& - Análisis de laboratorio \\
& - Registros de glucosa \\
\hline Apreciación & - Diagnósticos presuntivos \\
diagnóstica & - Diagnósticos definitivos \\
\hline Plan de tratamiento & - Recetas \\
& - Indicaciones y acuerdos \\
\hline
\end{tabular}

Adaptada de Gonzálz, (accesado 2020) ${ }^{9}$ y Mitsuishi, et al., $2016^{10}$.

mejorar la interacción y asegurar que no se pierden detalles se recomienda usar el método PSOAP (paciente, subjetivo, objetivo, apreciación diagnóstica, plan de tratamiento) $)^{9,10}$. Este método consiste en hacer la consulta de manera sistemática, tomando en cuenta las características del paciente con su lista de problemas y medicamentos. Hacer la evaluación subjetiva, tanto de los síntomas, como de las necesidades del paciente. La evaluación objetiva normalmente incluye la exploración física, la evaluación de análisis de laboratorio y de los registros de los diferentes dispositivos. De manera presencial siempre incluye la exploración física, pero a distancia, esta tendrá que ser limitada. Se debe documentar en el expediente electrónico lo que alcancemos a distinguir. Si la consulta telemática se realiza con videollamada, nos permite evaluar de manera general el estado de salud del paciente. Finalmente, se hace la apreciación diagnóstica, donde el médico, con toda la información recabada, hace sus diagnósticos presuntivos o definitivos y decide el plan de tratamiento, para lo cual realiza las recetas e indicaciones médicas (Tabla 1).

Al terminar la consulta se debe enviar la prescripción médica con firma digital de medicamentos e instrucciones y los análisis que se piden para la siguiente consulta. De preferencia, también debe mandar un resumen de las conclusiones y acuerdos de la consulta. En la tabla 2 se resumen los pasos de la consulta digital. 
Tabla 2. Pasos que seguir en una consulta a distancia

\begin{tabular}{|c|c|}
\hline 1. Solicitud de cita & $\begin{array}{l}\text { - Implementación de sistemas para agendar y confirmar cita: } \\
\text { - Sitio web } \\
\text { - Teléfono móvil (Ilamada, SMS, WhatsApp u otros) } \\
\text { - Correo electrónico } \\
\text { - Plataforma de expediente electrónico } \\
\text { - Sistema para el pago de la sesión a distancia, ya sea previo a la cita para confirmarla o al finalizar } \\
\text { - Servicios integrados en línea (Doctoralia }{ }^{\circledast}, \text { Nimblr }^{\circledast}, \mathrm{Nimbo}^{\circledR} \text {, etc.) }\end{array}$ \\
\hline 2. Preparación de consulta & $\begin{array}{l}\text { - Solicitar al paciente, recabar y enviar previo a la cita: } \\
\text { - Lista de medicamentos } \\
\text { - Signos vitales (de ser posible) } \\
\text { - Análisis de laboratorio } \\
\text { - Registros de glucosa } \\
\text { - Capacitación para la descarga de datos de dispositivos monitores, microinfusoras y otros }\end{array}$ \\
\hline 3. Consulta a distancia & Método PSOAP \\
\hline Conclusiones de la consulta & ío de recetas y acuerdos por vía digital \\
\hline
\end{tabular}

TELEMEDICINA UTILIZADA PARA EL DIAGNÓSTICO DE COVID-19

Un artículo interesante publicado a finales de marzo en el British Medical Journal describe los pasos necesarios para poder llevar a cabo una teleconsulta, de preferencia por videollamada, para valorar a los pacientes enfermos de COVID-19 y cómo guiarlos, dependiendo de los síntomas. Menciona que la mayoría de los pacientes pueden ser evaluados vía telemedicina, para decidir quiénes pueden ser tratados de manera ambulatoria, sin necesidad de ir a los centros de salud, y quiénes requieren atención hospitalaria. La ventaja de la videollamada es que permite ver si el paciente tiene dificultad respiratoria, aunque no cuente con un oxímetro. Cuenta con una infografía práctica, que sirve de guía para la teleconsulta realizada por los profesionales de la salud $^{11}$.

\section{PLATAFORMAS PARA TELEMEDICINA}

Con la pandemia del COVID-19, la mayoría de los expedientes médicos electrónicos que no contaban con eso han incorporado a su plataforma un sistema para llevar a cabo las consultas vía telemedicina. Estos permiten realizar la agenda de la cita, el cobro, la conexión con el paciente (mediante su misma plataforma, o vía Zoom ${ }^{\circledast}$ o GoogleHangouts ${ }^{\circledR}$ ) y la generación de recetas médicas digitales.

Cabe mencionar que existe una plataforma llamada Social Diabetes ${ }^{\oplus 12}$, que está diseñada específicamente para pacientes que viven con diabetes, y que cuenta además con una calculadora de bolo, para aquellos pacientes que se encuentran con esquema basalbolo de insulina y realizan conteo de carbohidratos.

\section{VENTAJAS Y DESVENTAJAS DE LA TELEMEDICINA}

Las ventajas de la telemedicina, incluyen que: a) no hay necesidad de transportarse, lo cual reduce el tiempo y costo indirecto de salud; b) nos permite conocer el entorno del paciente; c) si el paciente no recuerda la dosis de algún medicamento y/o vitamina, lo pueden revisar, ya que habitualmente va a realizar la teleconsulta en casa; d) permite que los pacientes se empoderen, al aprender a bajar los datos de sus dispositivos a la computadora y mandarlos al equipo de salud, y e) se puede hacer una consulta interdisciplinaria, con varios especialistas (médico endocrinólogo, nutriólogo, educador en diabetes) conectados al mismo tiempo, junto con el paciente y sus familiares o cuidadores, en caso necesario. 
Sin embargo, la principal ventaja de la telemedicina se encuentra en su poder social, puesto que permite que la alta especialidad pueda llegar a todos los sitios. McLendon, et al., en un estudio piloto dirigido a población rural con diabetes en descontrol, observaron en sus resultados una mejoría significativa en la hemoglobina glucosilada ( $\mathrm{HbA} 1 \mathrm{c})$, una reducción de visitas al hospital y disminución de costos en general ${ }^{13}$.

Las desventajas de la telemedicina incluyen que: a) no se puede realizar la exploración física, sin embargo, algunos detalles de esta sí se pueden evaluar y se deben de documentar en el expediente; b) pueden existir problemas con la conexión de internet, sobre todo si no se cuenta con una cable de ethernet o con audífonos; c) se debe tener cuidado con el uso de los datos personales y la privacidad; d) idealmente se debe contar con un consentimiento para llevar a cabo la teleconsulta, por parte del paciente, y e) las recetas digitales son válidas para la mayoría de los medicamentos, y en algunos casos para los antibióticos, pero aún no en México, para los medicamentos controlados. Un detalle que se debería resolver, ante la nueva realidad.

La telemedicina requiere la adquisición de nuevas habilidades en la comunicación, en el ejercicio de la clínica y en una nueva personalización del vínculo médico-paciente. Además de la procuración de protección legal para el médico y el paciente. La posibilidad de realización de teleconsultas internacionales también requerirá una discusión amplia a nivel global, que aún no está en la agenda política.

\section{¿POR QUÉ LA ENDOCRINOLOGÍA ES UNA ESPECIALIDAD PARTICULARMENTE COMPATIBLE CON LA TELEMEDICINA?}

La endocrinología, y en específico la diabetología, son especialidades ampliamente compatibles para el manejo con telemedicina. Si bien es cierto que el paciente debe ser visto presencialmente para su revisión anual (exploración física completa, incluyendo la revisión exhaustiva de pies, análisis de composición corporal, fondo de ojo), o cuando se encuentra sintomático, el seguimiento mensual o trimestral puede realizarse de manera remota, vía telemedicina sincrónica. En estas consultas, el objetivo es mejorar el manejo de la glucosa, la presión arterial y el perfil de lípidos. Para esto se necesitan mediciones objetivas, obtenidas del glucómetro, monitoreo continuo de glucosa, dispositivos para medición de presión arterial y análisis de laboratorio. Todos estos pueden ser enviados por vía digital al equipo de salud previo a la cita y ser analizados de manera sincrónica, en conjunto con el paciente y sus familiares, así como el personal pertinente del equipo de salud, para llegar a acuerdos de manejo de la enfermedad. Es por esto que, en un futuro, es muy posible que el manejo de los pacientes con enfermedades crónicas se haga de manera híbrida: presencial una vez al año o cuando el paciente se encuentra sintomático, y digital de manera periódica mensual o trimestralmente. Esto, necesariamente, va a disminuir los costos directos e indirectos (tiempo de traslado, tiempo en sala de espera) del manejo de las enfermedades crónicas.

Existe evidencia sobre el uso de telemedicina en diabetes que ofrece buenos resultados. Un estudio realizado por Ruth S. Weinstock sobre el uso de informática para telemedicina y educación en diabetes, durante cinco años, con el objetivo de realizar intervención y seguimiento a distancia en adultos mayores, demostró que hubo mejoría en el control glucémico, particularmente en población hispana (con niveles de $\mathrm{HbA1c}$ mayores al inicio del estudio), sugiriendo que la telemedicina tiene el potencial de optimizar el manejo en diabetes ${ }^{14}$.

Otro ejemplo es un estudio realizado en España, prospectivo a seis meses, llevado a cabo por Estmatjes, et al., en el cual se evaluó el impacto metabólico del seguimiento por medio de telemedicina en pacientes con diabetes tipo 1. Se comparó un grupo de intervención (mediante dos citas presenciales y cinco citas telemáticas) con un grupo control (mediante siete citas presenciales). Las variables estudiadas fueron costos, control metabólico, conocimiento de diabetes, calidad de vida y adherencia al tratamiento. Finalmente, como resultado, obtuvieron que el grupo de intervención requirió menos tiempo en consulta ( $823 \pm 645$ min vs. $353 \pm 222 \mathrm{~min}$ 
en grupo de intervención; $p<0.001$ ) y en cuanto a mejora en la $\mathrm{HbA1c}$, conocimiento, calidad de vida y adherencia al tratamiento, no hubo diferencias, concluyendo que el uso de citas telemáticas en pacientes con diabetes tipo 1 es una estrategia eficiente que ofrece resultados comparables a las citas presenciales ${ }^{15}$.

El estudio PLATEDIAN, publicado en Diabetes Care, con un diseño similar al previo, en pacientes con diabetes tipo 1, demostró que el uso de telemedicina era igual de eficaz y seguro que las citas presenciales, con impacto similar en el control glucémico, control y vigilancia de complicaciones y calidad de vida ${ }^{16}$.

La evidencia temprana muestra las ventajas de utilizar a la telemedicina, como una opción en el seguimiento de pacientes que viven con diabetes $y$ con excelentes resultados.

\section{MARCOS NORMATIVOS PARA EL USO} DE TELEMEDICINA O TELESALUD

En la Asamblea Mundial de la Salud del 2018, los gobiernos adoptaron por unanimidad una resolución en la que instaban a la OMS a elaborar una estrategia mundial sobre la salud digital para ayudar a los países a alcanzar la cobertura sanitaria universal, una estrategia que se examinará por la Asamblea Mundial de la Salud en el 2020. En abril del 2019, la OMS publicó recomendaciones para los países sobre maneras de mejorar la salud de la población y los servicios básicos mediante las tecnologías digitales (teléfonos móviles, tabletas y ordenadores). Las directrices muestran que los sistemas de salud deben responder a la creciente visibilidad y disponibilidad de la información, además, que se debe garantizar a los usuarios que no se revelarán sus datos y que el acceso a datos personales sobre temas delicados, como la salud sexual y reproductiva, no acarrea riesgos (OMS, 2019). Actualmente, México se encuentra en la creación de marcos legales y administrativos adecuados que consideren la incorporación y uso generalizado, cotidiano, de los servicios de telesalud ${ }^{17}$.
El programa de Acción de Telesalud 2007-2012 encuentra su fundamento legal en la Constitución Política de los Estados Unidos Mexicanos, donde el Estado reconoce como derecho de los mexicanos la protección a la salud y en la Ley General de Salud (LGS) que reglamenta las bases y modalidades para el acceso a los servicios de salud. La LGS en su artículo 7 establece que la coordinación del Sistema Nacional de Salud es responsabilidad de la Secretaría de Salud, a quien corresponde establecer y conducir la política nacional en materia de salud, en este caso en materia de telesalud, favoreciendo la participación de la comunidad y de las personas en el cuidado de su salud e impulsando permanentemente la actualización de las disposiciones legales requeridas.

El Programa de Acción Telesalud 2007-2012 encuentra su fundamento legal en: la LGS, el reglamento Interior de la Secretaría de Salud, la Ley sobre el Sistema Nacional de Asistencia Social y varias normas oficiales mexicanas aplicables. Con el Programa de Acción de Telesalud se buscó resolver la tarea pendiente de establecer una política pública que se adecúe a los marcos jurídicos y normativos y favorezca las inversiones estratégicas, logrando convertir la revolución digital en una oportunidad para el desarrollo del Sistema Nacional de Salud y de México².

Continuando en esta misma línea, se intentó trabajar en la construcción del Proyecto Norma Oficial Mexicana PROY-NOM-036-SSA3-2013 para la regulación de La Atención Médica a Distancia, la cual tenía como objetivo establecer los procedimientos que debe seguir el personal de salud que preste servicios de atención médica a distancia, sin embargo en diciembre 2015 se canceló el proyecto, ya que se determinó que el uso de las TIC como herramienta de apoyo y soporte para la medicina debe verse como una actividad integrada a la práctica clínica y no como actividad adicional, por ello no debe regularse de forma separada a los procedimientos médicos actuales ${ }^{18}$.

En 2017 se publicó la Cédula de Instrumentos Jurídicos aplicables a la práctica de telesalud en México, con actualización en abril del 2019, haciendo hincapié en que en la actualidad en México no existe normatividad específica para la materia, por tanto, el profesional de la salud que participa o realiza actividades en esta área debe observar las leyes que 
se aplican a la práctica médica ya existentes, siempre anteponiendo la seguridad del paciente, de sí mismo y finalmente la seguridad en la transmisión de la información. En dicha cédula se establece apegarse a la normatividad como cualquier otra actividad en el ámbito de salud. Finalmente, se comenta que la normalización de la telesalud presenta dificultades importantes para su total consolidación, y debe ser considerada como herramienta para fortalecer los servicios de salud y no como una solución.

En cuanto a la transmisión de información y datos personales, existe legislación en la Ley Federal de protección de datos personales en posesión de los particulares, la cual vela por el correcto tratamiento de la información personal, sobre todo en entornos digitales, además de fomentar las buenas prácticas y fortalecer los controles de protección de los datos personales fuera del ámbito gubernamental.

El reto actual para los tomadores de decisiones en la implementación y fortalecimiento de los programas de telesalud es precisamente sensibilizar al personal operativo en materia de los aspectos jurídicos, que les den una visión de lo que se puede y de lo que no se puede hacer con el uso de las tecnologías; otro reto es crear conciencia en el personal de salud de que la sobrerregulación traería confusión, que terminaría entorpeciendo o limitando el desarrollo de la telesalud o telemedicina en México ${ }^{19}$.

\section{FUTURO DE LA TELEMEDICINA}

En un futuro no muy lejano, todo lo que se requiera para una consulta médica va a poder ser transferido directamente desde los dispositivos del paciente al expediente electrónico médico. De manera automática, los archivos llegarán y se agruparán por fecha y descripción. Así mismo, los análisis de laboratorio se incorporarán automáticamente en una tabla de resumen, donde se podrán graficar para comparar los resultados de diferentes intervenciones, a lo largo del tiempo. Todo podrá ser visto en una sola plataforma, donde de forma simple se evaluará el manejo integral del paciente. Además, seguramente, con la ayuda de sistemas de inteligencia artificial se podrá ponderar el mejor tratamiento posible para cada paciente.

Actualmente, ya está disponible en algunos países un sistema de telecomunicación entre médico y paciente, que cuenta con un kit para medición de signos vitales y que facilita la exploración física del paciente (otoscopio, estetoscopio digital), y una App (TytoCare ${ }^{\circledast}$ ) que se comunica directamente con el médico y con su sistema de expediente electrónico, facilitando la teleconsulta ${ }^{19}$.

\section{CONCLUSIONES}

La telemedicina es útil en estos momentos en que el distanciamiento social es necesario; permite a los pacientes que viven con enfermedades crónicas continuar con su tratamiento y seguimiento. Los requerimientos mínimos para hacer telemedicina son: a) expediente médico electrónico; b) contar digitalmente y de manera previa a la consulta con la lista de medicamentos, los signos vitales, análisis de laboratorio y registros de glucosa; c) plataforma para videollamada, y d) recetas electrónicas.

La telemedicina llegó para quedarse, por lo que es importante que se regule de manera flexible, y que sea un servicio reembolsado de manera adecuada. Después de la pandemia por COVID-19, no se puede concebir el manejo de enfermedades crónicas, en especial la diabetes, sin la incorporación cotidiana de la telemedicina.

\section{FINANCIAMIENTO}

Los autores no recibieron patrocinio para llevar a cabo este artículo.

\section{CONFLICTO DE INTERESES}

Los autores declaran no tener conflicto de intereses alguno. 
RESPONSABILIDADES ÉTICAS

Protección de personas y animales. Los autores declaran que para esta investigación no se han realizado experimentos en seres humanos ni en animales.

Confidencialidad de los datos. Los autores declaran que en este artículo no aparecen datos de pacientes.

Derecho a la privacidad y consentimiento informado. Los autores declaran que en este artículo no aparecen datos de pacientes.

\section{BIBLIOGRAFÍA}

1. Zhu L, She ZG, Cheng X, Oin J, Zhang XJ, Cai J,et al. Association of blood glucose control and outcomes in patients with COVID-19 and pre-existing type 2 diabetes. Cell Metab. 2020 May 1. doi: 10.1016/j. cmet.2020.04.021. [Epub ahead of print]

2. Dabaghi A, Chávarri A, Torres A. Historia y filosofía de la medicina. Telemedicina en México. Anales Médicos. 2012;57(4):353-7.

3. Programa de Acción Específico 2007-2012. Telesalud. Primera edición. México: Secretaría de Salud.

4. Aparicio LA. Las TIC y la pandemia de influenza: Desafío para la Salud Pública. Rev Costarr Salud Pública. 2009;18:1-4.

5. Experiencias de Telemedicina en México. Sistema Nacional de Telesalud. Segunda edición. México: Secretaría de Salud; 2013.

6. Webster P. Virtual health care in the era of COVID-19. Lancet. 2020;395(10231):1180-1.
7. Qué es la telemedicina: definición y tipos [Internet]. Madrid, España: Clinic Cloud [31 de marzo del 2020]. Disponible en: https://clinic-cloud. com/blog/que-es-telemedicina-definicion-tipos/

8. Telehealth [Internet]. EE.UU.: U.S. Centers for Medicare \& Medicaid Services; 2020. Disponible en: https://www.medicare.gov/coverage/telehealth

9. Gonzálz D. Método PSOAP y ejemplo de nota médica [Internet]. Scribd [9 de mayo del 2020]. Disponible en: https://www.scribd.com/document/248988995/Metodo-PSOAP-y-Ejemplo-de-Nota-Medica

10. Mitsuishi F, Young JQ, Leary M, Dilley J, Mangurian C. The systems SOAP note: A systems learning tool. Acad Psychiatry. 2016;40(1):164-71.

11. Greenhalgh T, Koh GCH, Car J. Covid-19: a remote assessment in primary care. BMJ. 2020;368:m1182.

12. Brew-Sam N, Chib A. How do smart device apps for diabetes self-management correspond with theoretical indicators of empowerment? An analysis of App features. Int J Technol Assess Health Care. 2019;35(2):150 9.

13. McLendon SF, Wood FG, Stanley N. Enhancing diabetes care through care coordination, telemedicine, and education: Evaluation of a rural pilot program. Public Health Nurs. 2019;36(3):310-20.

14. Weinstock RS, Teresi JA, Goland R, Izquierdo R, Palmas W, Eimicke JP, et al. Glycemic control and health disparities in older ethnically diverse underserved adults with diabetes: five-year results from the Informatics for Diabetes Education and Telemedicine (IDEATel) study. Diabetes Care. 2011;34(2):274-9.

15. Esmatjes $E$, Jansà $M$, Roca $D$, Pérez-Ferre $N$, del Valle $L$, Martínez-Hervás $S$, et al. The efficiency of telemedicine to optimize metabolic control in patients with type 1 diabetes mellitus: Telemed study. Diabetes Technol Ther. 2014;16(7):435-41.

16. Ruiz de Adana MS, Alhambra-Expósito MR, Muñoz-Garach A, GonzalezMolero I, Colomo N, Torres-Barea I, et al. Randomized study to evaluate the impact of telemedicine care in patients with type 1 diabetes with multiple doses of insulin and suboptimal $\mathrm{HbA}_{1 c}$ in Andalusia (Spain): PLATEDIAN Study. Diabetes Care. 2020;43(2):337-42.

17. Cédula de Instrumentos Jurídicos aplicables a la práctica de la Telesalud en México [Internet]. México: Secretaría de Salud, Centro Nacional de Excelencia Tecnológica en Salud; 2019. Disponible en: https://www.gob. $\mathrm{mx} / \mathrm{salud} /$ cenetec/documentos/cedula-de-instrumentos-juridicos-aplicables-a-la-practica-de-la-telesalud-en-mexico

18. Marcos Normativos para el uso de la Telesalud [Internet]. México: Secretaría de Salud; 2015. Disponible en: http://www.cenetec.salud.gob.mx/ contenidos/telesalud/marco norma.html

19. McDaniel NL, Novicoff W, Gunnell B, Cattell Gordon D. Comparison of a novel handheld telehealth device with stand-alone examination tools in a clinic setting. Telemed J E Health. 2019;25(12):1225-30. 